FOLIA

Amazónico

Revista del Instituto de Investigaciones

de la Amazonía Peruana

\title{
EL CONTROL DE CALIDAD DE LA MADERA DE PLANTACIONES, UNA ALTERNATIVA PARA ALENTAR EL CULTIVO DE ÁRBOLES DE ESPECIES FORESTALES MADERABLES, ESTUDIO DE CASO DE Cedrelinga catenaeformis «TORNILLO»
}

\author{
Juan BALUARTE VÁSQUEZ ${ }^{1}$ \\ 1 Servicio Nacional Forestal y de Fauna Silvestre. Av. Javier Prado Oeste 2442. Magdalena del Mar, \\ Lima. Correo electrónico: jbaluarte@serfor.gob.pe
}

\section{RESUMEN}

En el presente estudio se ha comparado el turno óptimo de aprovechamiento de los árboles de plantaciones de «tornillo» con base en los incrementos medios anuales y los incrementos corrientes anuales en volumen de 1349 árboles, versus los resultados de los usos probables de la madera de plantaciones de la misma especie, basada en el estudio de las propiedades físicas, mecánicas y la trabajabilidad de la madera de diferentes edades, ubicadas en el Centro de Investigaciones Jenaro Herrera - CIJH. El primer estudio muestra que el turno óptimo de aprovechamiento silvicultural de «tornillo» ocurre a la edad de 13 años, cuando el leño de este aún no cuenta con la madurez suficiente comparada con la madera proveniente de bosques naturales. Alternativamente, el estudio de las propiedades físicas, mecánicas y trabajabilidad, recomienda el uso de la madera de «tornillo» a partir de los 15 años, reduciendo considerablemente el periodo de tiempo para que el leño llegue a la madurez, lo que permite inferir que la madera de «tornillo» puede ser utilizada antes de la maduración del leño. Esta información fue complementada con estudios de segunda fuente sobre los beneficios económicos y la estimación de carbono de la biomasa aérea de «tornillo» en plantaciones. Con este estudio se pretende contribuir a alentar el cultivo de árboles poniendo al alcance de los interesados el estudio de caso de esta especie y por otro lado, se alcanza recomendaciones para fomentar políticas para el cultivo y aprovechamiento de la madera de plantaciones.

PALABRAS CLAVE: Control de calidad, árboles maderables, política forestal, plantaciones forestales, fomento. 


\title{
QUALITY CONTROL OF PLANTATIONS TIMBER, AN ALTERNATIVE TO ENCOURAGE TREE GROWING OF TIMBER FOREST SPECIES, CASE STUDY OF Cedrelinga catenaeformis «TORNILLO»
}

\begin{abstract}
In the present study the optimal turn of use of the trees of «tornillo» plantations has been compared based on the annual average increments and the current annual increases in volume of 1349 trees, versus the results of the probable uses of the wood of plantations of the same species, based on the study of the physical, mechanical and workability properties of wood of different ages, located in the Jenaro Herrera Research Center - CIJH. The first study shows that the optimal turn of silvicultural use of "tornillo" occurs at the age of 13 years, when the wood of this still does not have sufficient maturity compared to wood from natural forests. Alternatively, the study of the physical, mechanical and workability properties, recommends the use of «tornillo» wood from the age of 15 , considerably reducing the period of time for the log to reach maturity, which allows inferring that the «tornillo» wood can be used before the wood matures. This information was complemented with second-source studies on the economic benefits and carbon estimation of aerial «tornillo» biomass in plantations. This study aims to help encourage the cultivation of trees by making the case study of this species available to those interested and, on the other hand, recommendations are made to promote policies for the cultivation and use of wood from plantations.
\end{abstract}

KEYWORDS: Quality control, timber trees, forest policy, forest plantations, promotion 


\section{INTRODUCCIÓN}

La superficie de bosques naturales del país es de aproximadamente 73 millones de hectáreas, siendo el segundo país con mayor extensión de bosques húmedos amazónicos después de Brasil (Deutsches Institut Für Entwicklungspolitik, 2015); no obstante, estas cifras no se condicen con el aporte de los bosques al desarrollo nacional; así la contribución del sector forestal al PBI es de $0.9 \%$ y la balanza comercial es negativa de menos 865 millones de Dólares USA (SERFOR, 2018).

Enlos últimos 27 años, los bosques naturales en el Perú han disminuido a un ritmo de $12 \%$ anual, al haber pasado de una superficie total de 73 millones de hectáreas a 63 millones de hectáreas (Deutsches Institut Für Entwicklungspolitik, 2015). La misma fuente indica que en el período 2001-2013 se ha observado pérdida de bosques a una tasa anual promedio de 113 mil hectáreas.

El 70\% de la deforestación está focalizada en cuatro departamentos amazónicos (San Martin, Loreto, Ucayali y Huánuco), equivalente a 7 millones de hectáreas aptas para reforestación. $\mathrm{Si}$ a esto adicionamos cerca de 7.5 millones de hectáreas para la forestación ubicadas en la región sierra (Rehab, www.cifor.org/rehab/_ref/ countris Perú/Brief-htm. Acceso 14/02/2019), estamos reconociendo que existe un enorme potencial de tierras con aptitud para el cultivo de árboles de especies maderables. El Plan Nacional Forestal y de Fauna Silvestre que se encuentra en etapa de construcción ha priorizado 2'568,654 ha de tierras aptas para el establecimiento de plantaciones, ubicadas en los departamentos andinos de Ancash, Apurímac, Huánuco, Junín y Pasco (SERFOR, 2018).

Mientras la deforestación de bosques avanza vertiginosamente, la recuperación de estas áreas es bastante lenta. Hasta el 2015 se acumuló 1.06 millones de hectáreas reforestadas, siendo las regiones de Cusco, Cajamarca, Ancash y Apurímac donde se ha sembrado más árboles (SERFOR, 2018).

La Ley Forestal y de Fauna Silvestre N ${ }^{\circ} 29763$ y su reglamento promulgado mediante Decreto Supremo $\mathrm{N}^{\circ}$ 020-2015-MINAGRI promueven la gestión de plantaciones forestales prioritariamente en zonas de recuperación con fines de producción forestal maderera con especies nativas y exóticas. Sin embargo, la siembra de árboles de especies maderables cuenta con numerosos obstáculos para su desarrollo, siendo el principal escollo su largo periodo biológico para la maduración del leño que a su vez permita obtener árboles con madera en condiciones similares a lo que provee el bosque natural, todo ello sumado a los altos costos de mantenimiento durante el periodo de establecimiento de los árboles.

La reducción de los bosques naturales en el mundo ha motivado que diversos países emprendan trabajos de investigación en silvicultura de especies forestales. Como producto de esas actividades se ha reportado una lista de 163 especies neotropicales para la producción de madera en condiciones de plantación entre las que está incluida Cedrelinga catenaeformis «tornillo» (Weaver, 1986).

Tornillo es, además una de las especies más estudiadas en el Perú en condiciones de plantación obteniendo resultados alentadores, constituyéndose en una especie promisoria para la reforestación de los bosques intervenidos de la Amazonía peruana (Claussi et al., 1992). Además, su caso puede servir para mostrar como el control de calidad de la madera puede contribuir a promover y masificar la siembra de árboles de especies forestales maderables, poniendo al alcance del estado peruano una alternativa práctica para promover el cultivo de árboles. 


\section{MATERIAL Y METODOS}

\section{DESCRIPCIÓN DE CEDRELINGA}

CATENAEFORMIS DUCKE "TORNILLO»

Cedrelinga catenaeformis crece naturalmente en las tierras altas de la Amazonía, en general prefiere los suelos de buen drenaje. En el Perú, la especie se encuentra en los departamentos de Amazonas, Huánuco, Junín, Loreto, Madre de Dios, Pasco, San Martín, Ucayali y Cuzco (Brako y Zarucchi, 1993).

C. catenaeformis pertenece a la familia botánica Fabaceae. La especie originalmente descrita fue incluida por Ducke en el género Piptadenia, posteriormente el mismo Ducke revisó la inestabilidad de la especie en el género Piptadenia reconsiderando a la especie y creando el nuevo género Cedrelinga (Spichiger, 1989).

La especie cuenta con una madera de densidad media; duramen marrón claro, lustroso, la albura es más claro; textura gruesa, semejante al cedro; grano recto a entrecruzado; fácil de trabajar con toda clase de herramientas de mano y máquinas, recibiendo buen acabado, firme y resistente; con buen comportamiento al secado al aire libre (Aróstegui, 1978).

La misma fuente, agrega que tradicionalmente la madera es utilizada en estructuras, construcciones livianas, civiles, navales, vigas, pisos, carrocerías, muebles ordinarios, carpintería de obra en general, implementos agrícolas, chapas de compensado, láminas decorativas, embalajes, moldes, prime decoraciones, adornos, bobinas y carretes.

\section{PROCEDIMIENTO PARA EL ANÁLISIS DE LA INFORMACIÓN}

La redacción de este documento se basó en el análisis de los resultados sobre crecimiento en diámetro y altura total de 1349 árboles de Cedrelinga catenaeformis «tornillo» proveniente del Centro de Investigaciones Jenaro Herrera -
$\mathrm{CIJH}$ que comprende mediciones multitemporales de 29 parcelas permanentes de crecimiento que abarcan el periodo de 1 a 27 años de edad reportados por Baluarte y Schwizer (2003). Estos resultados fueron contrastados con los usos probables de la madera producto del estudio de las propiedades físicas, mecánicas y la trabajabilidad de la madera de quince árboles de tornillo de plantaciones de 15, 24 y 29 años, ubicadas en el mismo CIJH que fueron publicados por Baluarte (2002).

El análisis de la información consistió en comparar el turno óptimo de aprovechamiento de los árboles de plantaciones de Jenaro Herrera, con base en los incrementos medios anuales y los incrementos corrientes anuales en volumen, versus los resultados de los usos probables de la madera de plantaciones de tornillo basada en la evaluación de la calidad de la madera a través de las propiedades supracitadas.

También se analizó las estadísticas sobre extracción de madera de Cedrelinga catenaeformis en el periodo 2000-2010 y los resultados del Inventario Nacional Forestal y de Fauna Silvestre de la ecozona Selva Baja, formulando con este último dato la estructura diamétrica de la densidad de árboles por hectárea y por clase diamétrica.

Adicionalmente, se analizó los resultados de publicaciones de segunda fuente para evaluar los beneficios económicos de la producción de madera y del secuestro de carbono en la biomasa aérea, datos que han servido para analizar la repercusión económica y social de la siembra de árboles en la población rural de la Amazonía peruana, en circunstancias en que la plantación de árboles surge como una alternativa para mitigar los efectos del cambio climático en el planeta.

Sobre la base de los resultados obtenidos, se proporcionan algunas recomendaciones para el fomento de políticas para el cultivo y 
aprovechamiento de árboles en plantaciones.

\section{RESULTADOS Y DISCUSIÓN}

\section{LA EXTRACCIÓN \\ DE CEDRELINGA \\ CATENAEFORMIS}

Cedrelinga catenaeformis se utiliza como madera aserrada a nivel comercial desde mediados del siglo pasado, sin embargo, el incipiente registro oficial en esa época no reportó los verdaderos valores de extracción. En los últimos 20 años con la mejora de los sistemas de control se observó la real magnitud del aprovechamiento de esta especie.

Es así como en el periodo 2000-2010, la madera de tornillo ocupó el primer lugar en aprovechamiento de madera aserrada con un total de 1'093,607.34 m³ y 1'421,280.18 m³ de madera en trozas (Ministerio de Agricultura, 2012). Esta última cifra representa aproximadamente 212,258 árboles de $65 \mathrm{~cm}$ de DAP promedio, talados en dicho periodo; no obstante, según los registros de la IUCN, la especie no está amenazada.

Según los resultados del Primer Panel del Inventario Nacional Forestal y de Fauna SilvestreINFFS (SERFOR, 2016) advierten la reducción de las poblaciones naturales en las clases diamétricas entre 50 y $70 \mathrm{~cm}$ y que constituyen los diámetros óptimos para el aprovechamiento comercial de árboles de esta especie (Figura $\mathrm{N}^{\circ} 1$ ), los árboles mayores de $70 \mathrm{~cm}$ por lo general presentan oquedades en la médula que limita su uso como madera aserrada (Jarama, 2004; Yépez y Linares, 2007), por esta razón, es frecuente encontrar en el bosque árboles de tornillo con DAP mayores de $80 \mathrm{~cm}$, la misma que se manifiesta en la Figura $\mathrm{N}^{\circ} 1$.

\section{CRECIMIENTO Y USOS PROBABLES DE CEDRELINGA CATENARFORMIS EN PLANTACIONES}

C. catenaeformis es una especie promisoria para el cultivo en plantación, sus incrementos en diámetro y altura total son superiores a $1 \mathrm{~cm} \mathrm{y}$ $1 \mathrm{~m}$ por año, respectivamente durante los diez primeros años (Claussi et al. 1992, Baluarte y Schwyzer, 2003), posteriormente, estos incrementos se ralentizan.

La evaluación del crecimiento en volumen de esta especie en condiciones de plantación en Jenaro Herrera desvela que el punto óptimo de incremento en volumen para el aprovechamiento silvicultural de los árboles de tornillo ocurre a los 13 años cuando los árboles alcanzan $22.29 \mathrm{~m}^{3}$ / ha (Figura $\mathrm{N}^{\circ}$ 2) (Baluarte y Schwyzer, 2003), sin embargo, a esta edad el leño de la madera aún no está lo suficientemente madura para los usos tradicionales mencionados por Aróstegui (1978).

En el Centro de Investigaciones Jenaro Herrera, los estudios sobre la silvicultura de tornillo fueron complementados con la evaluación de la calidad de la madera a través de estudios de las propiedades físico-mecánicas y ensayos de 


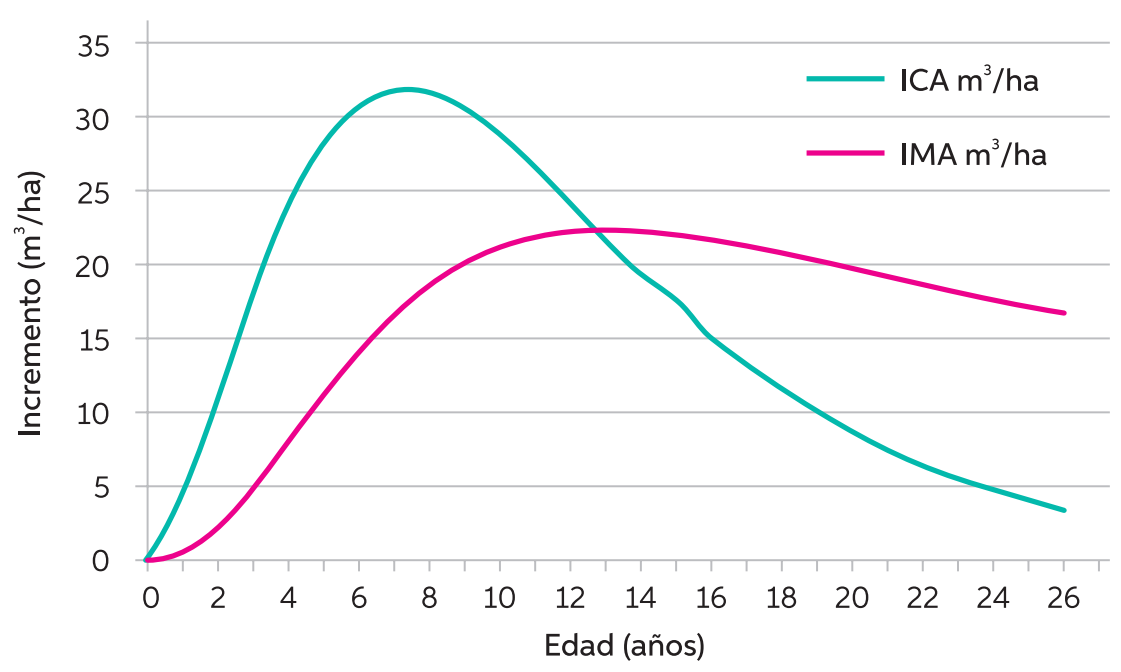

Figura 2. Turno óptimo de aprovechamiento de los árboles de tornillo en plantaciones de Jenaro Herrera con base al incremento medio anual e incremento corriente anual en volumen.

Tabla 1: Usos probables de la madera tornillo de plantaciones de diferentes edades en Jenaro Herrera, río Ucayali

\begin{tabular}{|c|c|c|c|c|c|}
\hline \multirow[b]{2}{*}{ Especie } & \multirow{2}{*}{$\begin{array}{c}\text { Edad } \\
\text { de la } \\
\text { plantación } \\
\text { (años) }\end{array}$} & \multicolumn{2}{|c|}{$\begin{array}{c}\text { Construcción de } \\
\text { Viviendas }\end{array}$} & \multirow{2}{*}{$\begin{array}{l}\text { Encofra- } \\
\text { dos }\end{array}$} & \multirow{2}{*}{ Mueblería } \\
\hline & & $\begin{array}{l}\text { Estruc- } \\
\text { tura }\end{array}$ & $\begin{array}{c}\text { Carpintería } \\
\text { de obra }\end{array}$ & & \\
\hline \multirow{3}{*}{ Tornillo } & 15 & - & $\mathbf{x}$ & - & $\mathbf{x}$ \\
\hline & 24 & - & - & $x$ & - \\
\hline & 29 & - & $x$ & - & $x$ \\
\hline
\end{tabular}

Fuente: Baluarte (2002)

trabajabilidad de la madera de plantaciones de diversas edades de desarrollo.

$\mathrm{Al}$ respecto, este estudio concluye que la madera de tornillo puede ser utilizada a partir de 15 años, recomendando el uso probable en mueblería como: bancos, mesas, reposteros, armarios y carpetas (tabla $\mathrm{N}^{\circ} 1$ ) (Baluarte 2002); acortando de esta manera el periodo de espera para lograr la maduración del leño de la madera de esta especie.

Los usos sugeridos en la tabla $\mathrm{N}^{\circ} 1$, resultado del control de calidad de la madera de plantacio- nes a partir de los 15 años, es muy cercana al turno óptimo de aprovechamiento silvicultural, convirtiéndose de esta manera en una herramienta adicional muy útil para la toma de decisiones en la gestión de plantaciones de especies forestales maderables. El costo financiero que genera el mantenimiento de una plantación por encima del turno óptimo de aprovechamiento silvicultural se eleva exponencialmente en caso la plantación se establezca con fondos provenientes de entidades financieras. Esta misma metodología se puede aplicar a todas las especies forestales maderables que se cultivan en condiciones de plantación.

Actualmente, los estudios de las propiedades mecánicas de la madera han evolucionado de métodos destructivos a la utilización de sensores de sonido que captan longitudes de onda que permiten determinar las características mecánicas de la madera de árboles en pie, sin necesidad de abatirlos, haciendo más atractiva esta actividad desde el punto de vista ambiental.

\section{EL CULTIVO DE ÁRBOLES DE CEDRELINGA CATENAEFORMIS Y SU CONTRIBUCIÓN SOCIO ECONÓMICA AL PAÍS}

El cultivo de árboles de tornillo en la Amazonía peruana puede contribuir a la generación de empleo e ingresos en el ámbito rural del país, convirtiéndose en una alternativa productiva para la jubilación de miles de pobladores que practican la agroforestería.

La relación beneficio-costo por hectárea de una plantación de tornillo de 30 años arroja 
resultado positivo de 1.32 de madera en trozas, es decir, por cada S/. 1.00 que se invierte, es posible obtener S/. 1.32 como devolución (Álvarez y Ríos, 2009); sin embargo, de acuerdo con las reglas de la economía, cuanto mayor es el valor agregado del producto, esta relación será mucho mayor.

El cultivo de árboles de especies maderables también contribuye a mitigar los gases de efecto invernadero. En ese contexto, las plantas, a través de la fotosíntesis, absorben el $\mathrm{CO}_{2}$ de la atmosfera (uno de los 6 principales gases que intervienen en el efecto invernadero) y lo fijan como carbono en su biomasa (madera, hojas, ramas, raíces). La permanencia de carbono en la biomasa vegetal depende del ciclo de vida de las plantas. Los árboles y demás especies leñosas pueden almacenar carbono por años en su madera (Avendaño, 2006).

Estudios realizados en el Centro de Investigaciones Jenaro Herrera sobre captura de carbono en plantaciones de tornillo de treinta y cinco años revela que la biomasa aérea y necromasa estimada es de 365 t/ha para calidad de sitio alta y $310.15 \mathrm{t} / \mathrm{ha}$ para sitio de calidad media, lo que equivale a 182 y $155 \mathrm{tC} / \mathrm{ha}$, respectivamente (Saldaña, 2004), que representa aproximadamente entre US \$1,224.5 a \$1,437.8 por hectárea, monto que suma a los bienes tangibles antes mencionados.

En ese sentido, la producción de madera de tornillo y la prestación de servicios ambientales podría contribuir a la economía de la población rural generando ingresos por la comercialización de la madera y el pago por servicios ambientales. Esta actividad económica bien organizada en el ámbito rural coadyuvaría a mejorar las condiciones económicas de la población rural asentándolas en el lugar, evitando de esta manera la migración de la población rural a las ciudades y sus repercusiones negativas que conlleva en la economía del país.

\section{CONCLUSIONES}

El cultivo de árboles de especies forestales es por lo general una actividad que implica un largo periodo de espera para la devolución de la inversión, debido a las características biológicas intrínsecas de los árboles de especies maderables, situación que las convierte en poco atractivas frente a otros cultivos con menor periodo de aprovechamiento y devolución de la inversión.

Por lo general, el silvicultor utiliza el turno silvicultural y el turno financiero como herramientas al momento de tomar decisiones para el aprovechamiento de la madera de plantaciones; sin embargo, el turno silvicultural por lo general ocurre muy temprano cuando el leño aún está un poco verde como el caso estudiado; en este contexto, el costo de mantener una plantación por encima del turno óptimo de aprovechamiento, se eleva exponencialmente generando altos costos si el dinero ha sido gestionado a través del sistema financiero.

Ante esta situación, el control de calidad de la madera de plantaciones surge como una alternativa adicional en la gestión de plantaciones de especies forestales maderables, poniendo al alcance de los pobladores rurales y de los silvicultores los usos probables de la madera de plantaciones de diferentes edades con bases sólidas y científicas - «turno tecnológico».

El control de calidad de la madera que se produce en plantaciones puede contribuir a promover y masificar la siembra de árboles de especies forestales maderables, debido a que reduce el período de aprovechamiento de los árboles haciendo más atractiva esta actividad entre los pobladores rurales y los inversionistas.

La reducción del periodo de aprovechamiento de árboles de plantaciones no solo incentivará su cultivo sino también generará mayor empleo en el ámbito rural fijando a las pobladores rurales, 
evitando de esta manera la migración a las grandes ciudades. Además, el cultivo de árboles debe ir acompañado de políticas para la generación de mayor valor agregado de los productos.

Por otro lado, el control de calidad de la madera de plantaciones por métodos no destructivos impulsará la formación de pequeñas empresas especializadas en este rubro, generando mayor empleo en el sector privado.

El estudio de caso aquí reportado sirve como ejemplo para aplicar a cualquier otra especie forestal maderable cuya población natural haya sido severamente afectada por la extracción selectiva de acuerdo con los registros del INFFS.

La plantación de árboles puede contribuir a mejorar las condiciones socio económicas de los pobladores rurales que practican la agroforestería, convirtiéndose en un fondo de jubilación para el retiro digno de los pobladores rurales.

\section{RECOMENDACIONES PARA FOMENTAR POLÍTICAS PARA EL CULTIVO DE ÁRBOLES}

- Promover políticas de innovación que incentiven el control de calidad de la madera de plantaciones mediante métodos no destructivos que permitan la utilización de la madera de plantaciones jóvenes, haciendo atractiva esta alternativa productiva para los pobladores rurales e inversionistas.

- Fomentar políticas de innovación para generar mayor valor agregado a la madera proveniente de plantaciones a través de la producción de artículos utilitarios para fomentar el consumo interno y externo.

- Fomentar la creación de centros de formación artesanal de naturaleza pública en las capitales de distritos rurales dedicados a la enseñanza, transformación y generación de mayor valor agregado de la madera proveniente de plantaciones.
- Desarrollar estrategias de mercadeo para ofertar los productos y artículos utilitarios fabricados con madera proveniente de plantaciones.

- Normar el registro de empresas dedicadas al control de calidad de la madera de plantaciones con estándares ambientales estrictos y con personal altamente calificado para el control calidad de la madera.

\section{AGRADECIMIENTOS}

Al Instituto de Investigaciones de la Amazonia Peruana-IIAP por proporcionar los datos decampo de las parcelas permanentes de crecimiento y los resultados de los estudios de las propiedades físicas, mecánicas y trabajabilidad de la madera de plantaciones de Cedrelinga catenaeformis ubicados en el campo experimental del Centro de Investigaciones Jenaro Herrera. Al Instituto Nacional de Innovación Agraria-INIA y a Andreas Schwyzer del Swiss Federal Institute-WSL (Suiza) coautor del informe sobre crecimiento y productividad de «tornillo» en plantaciones, a la Universidad Nacional Agraria por el estudio de las características físicas, mecánicas y trabajabilidad de la madera de la misma especie.

\section{REFERENCIAS BIBLIOGRÁFICAS}

Álvarez, L. y Ríos, S. 2009. Evaluación económica de plantaciones de tornillo, Cedrelinga catenaeformis en el departamento de Loreto. Avances Económicos $\mathrm{N}^{\circ} 10$. Instituto de Investigaciones de la Amazonía Peruana. Iquitos, Perú. $37 \mathrm{pp}$.

Aróstegui, A. 1978. Estudio tecnológico de maderas del Perú (Zona Pucallpa); Vol I Características tecnológicas y usos de la madera de 145 especies del país. Ministerio de Agricultura, Universidad Nacional Agraria, 
La Molina. $483 \mathrm{pp}$.

Avendaño, H. D. M. 2006. Determinación de ecuaciones alométricas para estimar biomasa y carbono en Abies religiosa (H. B. K.) Schl. et Cham., en Tlaxcala, México. Tesis Profesional. División de Ciencias Forestales. UACH. Chapingo, Texcoco, Estado de México. 66 pp.

Baluarte, J. 2002. Implicancias silviculturales de los estudios tecnológicos de maderas de plantaciones de tres especies forestales del Centro de Investigaciones Jenaro Herrera, Loreto. Instituto de Investigaciones de la Amazonía Peruana-IIAP. En: VIII Congreso Nacional Forestal y IV Asamblea Nacional del Capítulo de Ingenieros Forestales. Iquitos 6-8 noviembre 2002. Perú. 15 pp.

Baluarte, J. y Schwyzer, A. 2003. Crecimiento y Productividad de Cedrelinga catenaeformis Ducke «tornillo» en plantaciones. Informe anual 2003. Estación Experimental San Roque, Instituto Nacional de Innovación Agraria. Iquitos. 20 pp.

Brako, L. y Zarucchi, J. 1993. Catálogo de las Angiospermas y Gimnospermas del Perú. Missouri Botanical Garden. St. Louis, Missouri, USA. 1286 pp.

Claussi, A., D. Marmillod y J. Blaser. 1992. Descripción silvicultural de las plantaciones forestales de Jenaro Herrera. IIAP. Iquitos, Perú. 332 pp.

Deutsches Institut Für Entwicklungspolitik, German Development Institute, Global Green Growth Institute, SERFOR. 2015. Interpretación de la dinámica de la deforestación en el Perú y lecciones aprendidas para reducirla. Documento de Trabajo. Lima. $40 \mathrm{pp}$.

Jarama, L. 2004. Evaluación de la enfermedad «Pudrición medular» de la especie Cedrelinga catenaeformis, Ducke"tornillo" en plantaciones forestales del Centro de Investigaciones Jenaro Herrera (CIJH). Instituto de Investigaciones de la Amazonía Peruana - IIAP. lquitos, Perú. $181 \mathrm{pp}$.

Ministerio de Agricultura. 2012. Estadística Forestal del Perú 2000-2010. Dirección Forestal y de Fauna Silvestre. Lima. 186 pp.

REHAB. Degradación forestal y rehabilitación en Perú. www.cifor.org/rehab/_ref/countris Perú/Brief-htm. Acceso 14/02/2019

Saldaña. P. 2004. Estimación de la biomasa de Cedrelinga catenaeformis Ducke (Tornillo) y Simarouba amara Aublet (Marupá) en plantaciones forestales del Centro de Investigaciones Jenaro Herrera. Tesis para optar el título de Ingeniero Forestal UNAP. Iquitos. $52 \mathrm{pp}$.

SERFOR. 2016. Primer Informe Parcial del Inventario Nacional Forestal y de Fauna Silvestre. Lima. 271 pp + anexos

SERFOR. 2018. Plan Nacional Forestal y de Fauna Silvestre; conocimiento integral de la realidad del sector forestal - Primera Fase. Borrador de Trabajo. Lima. 53 pp.

Spichiger, R.; Méroz, J.; Loizeau, P.A. y Stutz de Ortega, L. 1989. Contribución a la flora de la Amazonía peruana; los árboles del Arboretum Jenaro Herrera. Conservatorio y Jardín Botánicos de la ciudad de Ginebra, Organización Suiza para el Desarrollo y la Cooperación, Instituto de Investigaciones de la Amazonía Peruana. Vol. 1. Genéve, Suiza. $359 \mathrm{pp}$.

Yepez, F. y Linares, C. 2007. Rendimiento de trozas de trozas aserradas de Cedrelinga catenaeformis Ducke obtenidas del raleo silvicultural de plantaciones en Jenaro Herrera, Loreto, Perú. Folia Amazónica V16 (1-2): 115120.

Weaver, P.L. 1986. Enrichment plantings in Tropical America. In: Conference management of the forest of Tropical America: Prospects and Tecnologies. San Juan Puerto Rico. Proceeding: 259-278.

Recibido: 27 de febrero de 2019 Aceptado para publicación: 13 de junio de 2019 Esta obra está bajo una Licencia Creative Commons Atribución-NoComercial-SinDerivar 4.0 Internacional. 